\title{
Analysis of Changes in Retinal Thickness in Type 2 Diabetes without Diabetic Retinopathy
}

\author{
Jing Jiang, ${ }^{1,2}$ Yan Liu, ${ }^{1,3}$ Yingchao Chen, ${ }^{4}$ Bo Ma, ${ }^{1}$ Yiwen Qian, ${ }^{2}$ Zhenzhen Zhang, \\ Dongqing Zhu $\mathbb{D}^{1},{ }^{1}$ Zhiliang Wang $\mathbb{D}^{2},{ }^{2}$ and Xiaofang $X u \mathbb{D}^{1,3}$ \\ ${ }^{1}$ Department of Ophthalmology, Ninth People's Hospital, Shanghai JiaoTong University School of Medicine, Shanghai, China \\ ${ }^{2}$ Department of Ophthalmology, Huashan Hospital of Fudan University, Shanghai, China \\ ${ }^{3}$ Shanghai Key Laboratory of Orbital Diseases and Ocular Oncology, Shanghai, China \\ ${ }^{4}$ Department of Endocrinology, Shanghai Ninth People's Hospital, Shanghai Jiaotong University School of Medicine, Shanghai, China
}

Correspondence should be addressed to Zhiliang Wang; ophwzl@163.com and Xiaofang Xu; xuxu0139@hotmail.com

Received 29 June 2017; Revised 22 September 2017; Accepted 30 October 2017; Published 25 February 2018

Academic Editor: Amany Tawfik

Copyright $\odot 2018$ Jing Jiang et al. This is an open access article distributed under the Creative Commons Attribution License, which permits unrestricted use, distribution, and reproduction in any medium, provided the original work is properly cited.

Objective. To examine the changes in retinal thickness of patients with diabetes without DR. Designs. A randomization, crossover, retrospective practice. Participants. 43 diabetic patients and 43 ethnic-, age-, and sex-matched controls. Methods. Full retinal thicknesses of ten areas were assessed using spectral domain optical coherence tomography. Confounding variables, such as age, gender, and glycated haemoglobin (HbAlc) level, were assessed by regression analysis. Main Outcome Measures. Mean retinal thickness of ten areas. Results. The mean thickness of the fovea was $215.8 \pm 18.9 \mu \mathrm{m}$ in the diabetes group and $222.0 \pm 18.6 \mu \mathrm{m}$ in the control group $(p=0.04)$. The mean thickness of the temporal parafovea was $319.9 \pm 16.7 \mu \mathrm{m}$ in the diabetes group and 326.0 $\pm 14.4 \mu \mathrm{m}$ in the control group $(p=0.01)$. The mean thickness of the temporal perifovea was $276.4 \pm 27.9 \mu \mathrm{m}$ in the diabetes group and $284.8 \pm 17.4 \mu \mathrm{m}$ in the control group $(p=0.02)$. There were no significant differences in retinal thickness between groups in other areas $(p>0.05)$. Regression analysis revealed that decreased retinal thickness of the temporal perifovea was associated with a higher HbAlc level $(>8.7 \%)(p=0.04)$. Conclusion and Relevance. Subtle structural changes in the retina may occur in diabetes without DR. Decreased retinal thickness appeared to begin in the fovea and temporal areas. A high HbAlc level was the main factor influencing retinal thickness.

\section{Introduction}

Diabetes is a disease characterized by metabolic dysregulation. It occurs worldwide, and the number of people with diabetes is estimated to increase dramatically from 171 million in 2000 to 366 million in 2030 [1]. One-third of diabetic people develop some degree of diabetic retinopathy (DR), which has become the leading cause of vision loss in working-age adults [2]. It is important to detect the early signs of DR to facilitate timely monitoring and referral.

Clinical features of DR are undetectable at early stages. Traditional methods for evaluating DR, including slit-lamp biomicroscopy and stereo fundus photography, are relatively insensitive to small pathological changes in the retina. In addition, highly sensitive fluorescein angiography is invasive and not suitable for repeated examination. Optical coherence tomography (OCT) is a rapid, noninvasive, and useful imaging technology for cross-sectional and tomographic imaging in biological tissues, which is especially useful for quantitative and qualitative assessment of macula $[3,4]$. OCT can provide objective documentation of retinal structural changes in eyes with DR even when the changes are not evident through slitlamp biomicroscopy or angiography $[5,6]$.

Several studies have elucidated changes in the retinal thicknesses in patients with diabetes. Increases or decreases in retinal thickness have been reported in diabetes with or without DR, respectively, and various mechanisms have been proposed to be responsible for these changes [7-9]. The purpose of this study was to quantify the differences in retinal thickness between healthy individuals and individuals with 
diabetes without DR. In addition, our study evaluated the association of retinal thickness with age, gender, duration of diabetes, and glycated haemoglobin (HbA1c) levels.

\section{Methods}

This was a single-centre, retrospective cross-sectional case series performed at the Department of Ophthalmology, Ninth People's Hospital, Shanghai JiaoTong University, School of Medicine, from February 1, 2016, to July 15, 2016. The study was approved by the institute's ethics committee. Informed consent was provided by each subject for participation in the study.

2.1. Subjects. A total of 86 eyes of 43 patients with type 2 diabetes mellitus (group A) and the same number of samples in healthy subjects (group B, control group) were recruited. All participants underwent a comprehensive ophthalmologic examination consisting of visual acuity assessment (best corrected visual acuity [BCVA]), intraocular pressure (IOP) assessment, slit-lamp biomicroscopy combined with retinoscope, and fundus fluorescein angiography (FFA). The levels of peripheral fasting blood glucose (Portable Blood Glucose Meters; Johnson OneTouch Ultra; Johnson \& Johnson Co, NJ, USA) and glycated haemoglobin A1C (HbA1c) were also simultaneously measured. The measurement method of $\mathrm{HbAlc}$ was high-pressure liquid chromatography (HPLC; Bio-rad Variant II; Bio-rad Co, CA, USA). This detection method is relatively accurate and stable, which has been widely used in routine screening for diabetes in China $[10,11]$.

For group A, an eye was eligible if it met the following criteria: (1) definite diagnosis of type 2 diabetes; (2) no DR (Early Treatment Diabetic Retinopathy Study level 10) on the basis of clinical exam and FFA; (3) no history of major ocular surgery or treatment, such as panretinal photocoagulation (PRP); (4) no macular pathology, such as epimacular membrane (EMM), vitreomacular traction (VMT), and age-related macular degeneration (AMD) found during clinical examination; and (5) no refractive errors or a refractive error less than $\pm 3.0 \mathrm{D}$. For group $\mathrm{B}$, the inclusion criteria were the same except that the subjects had no history of diabetes.

2.2. Optical Coherence Tomography Imaging. The retinal images were obtained using a Heidelberg Spectralis OCT instrument (wavelength: $870 \mathrm{~nm}$; Heidelberg Engineering Co, Heidelberg, Germany) with version 6.0 software. Heidelberg Eye Explorer software (version 1.9.10.0, Heidelberg Engineering Co, Heidelberg, Germany) was used to perform measurements.

Retinal thickness was defined as the height from the vitreoretinal interface to the retinal pigment epithelium (RPE) (Figure 1) [7, 12]. The macular region, with the exception of the fovea, was divided into three zones (centre, parafovea, and perifovea) with three concentric circles whose diameters were 1,3 , and $6 \mathrm{~mm}$, respectively. Then, the parafovea and perifovea zones were further subdivided into four quadrants with two diagonal lines. Finally, in this study, a total of ten areas (fovea, centre, superior parafovea, superior perifovea, inferior parafovea, inferior perifovea, nasal parafovea, nasal perifovea, temporal parafovea, and temporal perifovea) were available for analysis. Firstly, the zones were partitioned automatically by using Stratus OCT software (version 6.0; Heidelberg Engineering Co, Heidelberg, Germany); then it would be artificially adjusted according to actual situation of the picture by the same examiner (Dr. Jing Jiang). A schematic of the examined zones is presented in Figure 2.

The retinal thickness was measured along 25 horizontal lines, each of which was $6 \mathrm{~mm}$ long and centred at the fovea. Thirty frames were averaged together with the aid of eye tracking, and images with a quality score $\geq 25$ were selected. The lines crossing the foveal region were automatically calculated and converted to foveal thickness by using Stratus OCT software (version 6.0; Heidelberg Engineering Co, Heidelberg, Germany). For the remaining regions, average retinal thickness was calculated as the weighted average of all thickness measurements in the region (Figure 3). Data were then reassessed by two blinded independent examiners (Dr. Jing Jiang and Dr. Yan Liu). A third blinded investigator (Dr. Xiaofang $\mathrm{Xu}$ ) made the final decision if disagreements and discrepancies in the assessments arose.

2.3. Statistical Analysis. Statistics were calculated using SPSS software (IBM SPSS, Version 22.0, IBM Corporation, Armonk, NY). Continuous variables, such as age, HbAlc level, and retinal thickness, are presented as the means $\pm \mathrm{SD}$. The chi-square test and two-sample $t$-test were performed to evaluate the differences between the study groups. The Pearson correlation coefficient was used to assess the correlation between any two nonnormally distributed variables. All $p$ values reported were two-tailed, and $p<0.05$ was considered to be statistically significant in this study.

\section{Results}

In total, 86 subjects (with 172 eyes) were recruited in this study. The average age of all subjects was $64.0 \pm 7.0$ years (range 45-80 years), and the male/female ratio was 32/54. A summary of the clinical parameters of participants in the two groups is provided in Table 1. Except for the history of diabetes and the higher HbAlc levels in group A, no further significant differences were observed between the two groups.

3.1. Changes in Retinal Thickness in Multiple Areas. Overall, the retinal thickness was thinnest in the foveal area, followed by the central area, in all subjects. The parafoveal areas were thicker than the perifoveal areas, and the nasal areas were thicker than the temporal areas. On average, the retinal thicknesses in all ten areas were thinner in group A than in group B. However, only the differences in the foveal, temporal parafoveal, and temporal perifoveal areas were statistically significant $(p=0.04,0.01$, and 0.02 , resp.). Table 2 presents a summary of the retinal thickness of the ten areas in both groups. 

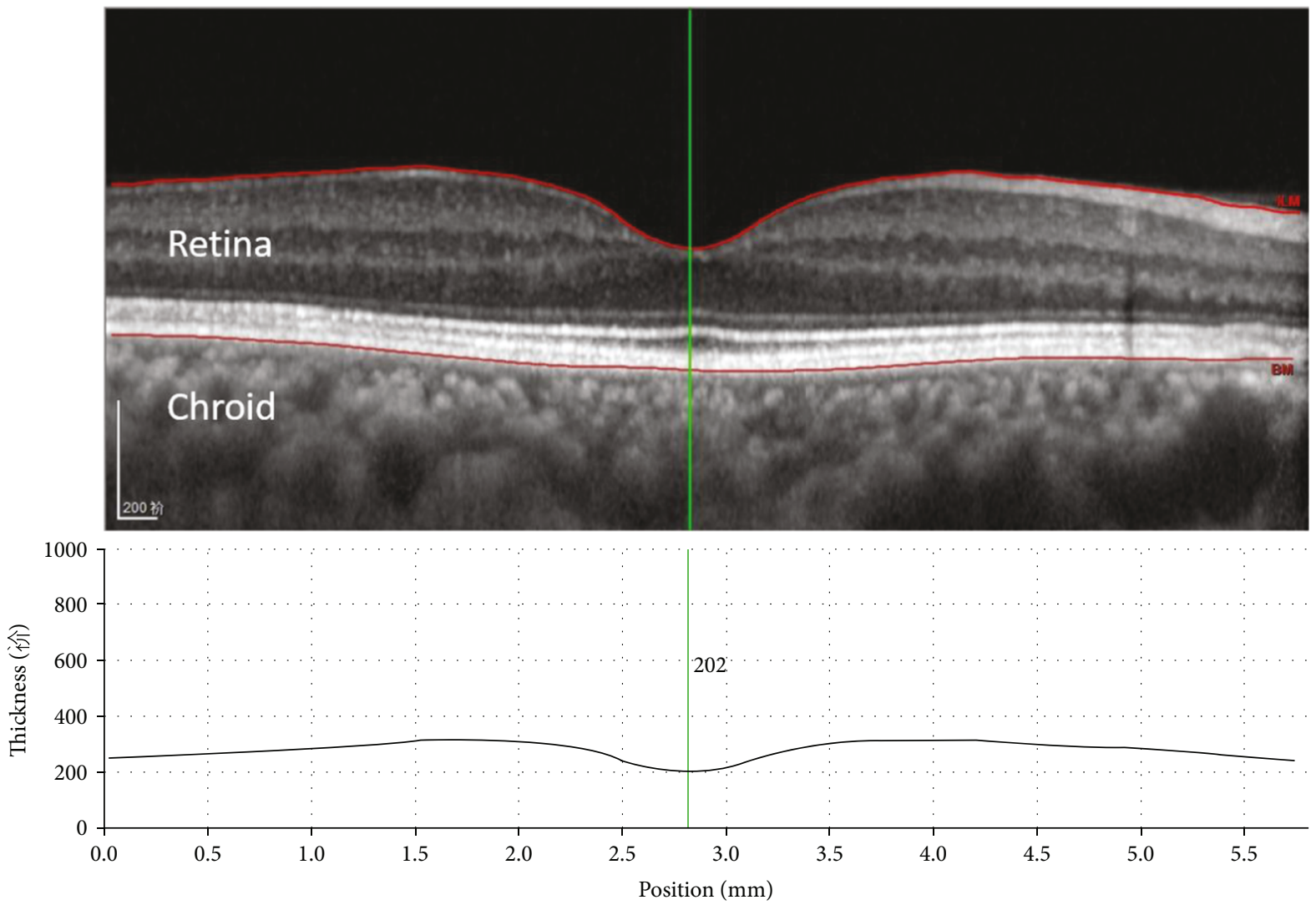

FIGURE 1: Enhanced depth imaging spectral domain optical coherence tomography (EDI-SD OCT) using Heidelberg Spectralis. The scan reveals normal retinal and choroidal anatomy. The foveal retinal thickness was determined on the basis of the distance between the two segments (red lines), which was measured in the "thickness profile" window by using the thickness diagram at the selected targets (the green line).

3.2. Factors Affecting the Thickness of the Retina. The duration of diabetes is a major factor that influences the progression of DR. In this study, the thickness of temporal areas showed a trend towards thinning with an increased duration of diabetes $(r=-0.07,-0.20)$, but the differences were not statistically significant $(p>0.05)$.

The HbA1c level was another important factor affecting DR. To further explore the potential effect of HbAlc levels on retinal thickness, we divided the patients in group A into 2 subgroups according to their HbA1c levels. Group A1 $\left(\mathrm{G}_{\mathrm{Al}}\right)$ included subjects with an HbA1c level $\leq 8.7 \%$, and group $2\left(\mathrm{G}_{\mathrm{A} 2}\right)$ included subjects with an $\mathrm{HbA} 1 \mathrm{c}$ level $>8.7 \%$. After correction for age and sex, the Pearson correlation analysis revealed that only the temporal perifoveal thickness exhibited a negative correlation with HbA1c level $(r=-0.31, p=0.04)$, thus indicating that an increased $\mathrm{HbAlc}$ level contributed to decreased retinal thickness (Table 3).

\section{Discussion}

In this study, we examined retinal thickness in normal and type 2 diabetic individuals with no DR using SD-OCT and analyzed possible factors affecting retinal thickness. Our results indicated that thicknesses in the foveal and temporal (parafovea and perifovea) areas were significantly decreased in diabetic individuals with no visible sign of DR compared with normal controls. A thinning of the retina in the temporal perifovea area was associated with an increased $\mathrm{HbA1c}$ level $(>8.7 \%)$.

In humans, diabetes can be classified into insulindependent diabetes mellitus (type 1 diabetes mellitus, T1DM) and noninsulin-dependent diabetes mellitus (type 2 diabetes mellitus, T2DM) according to pathogenesis [13]. Although the pathomechanism and prevalence populations of two types are totally different, retinal thickness and structure between T1DM and T2DM did not observe statistically significant difference after controlling for age, sex, duration of diabetes, and HbA1c levels. Generally speaking, T1DM has a prolonged course while T2DM is slightly older. Therefore, the potentially confounding effect of these variables may assess retinal thickness [14]. In our research, all patients were T2DM.

Changes in retinal thickness caused by diabetes are not fully understood. Previous studies have found decreased retinal thickness in diabetes with minimal or no DR compared with retinal thickness in nondiabetic individuals $[15,16]$. In contrast, other researchers have observed a tendency towards increased retinal thicknesses in individuals with advanced DR $[17,18]$. Our study provided an overall profile of retinal thickness in individuals with diabetes without detectable signs of DR. We observed that retinal thickness was 


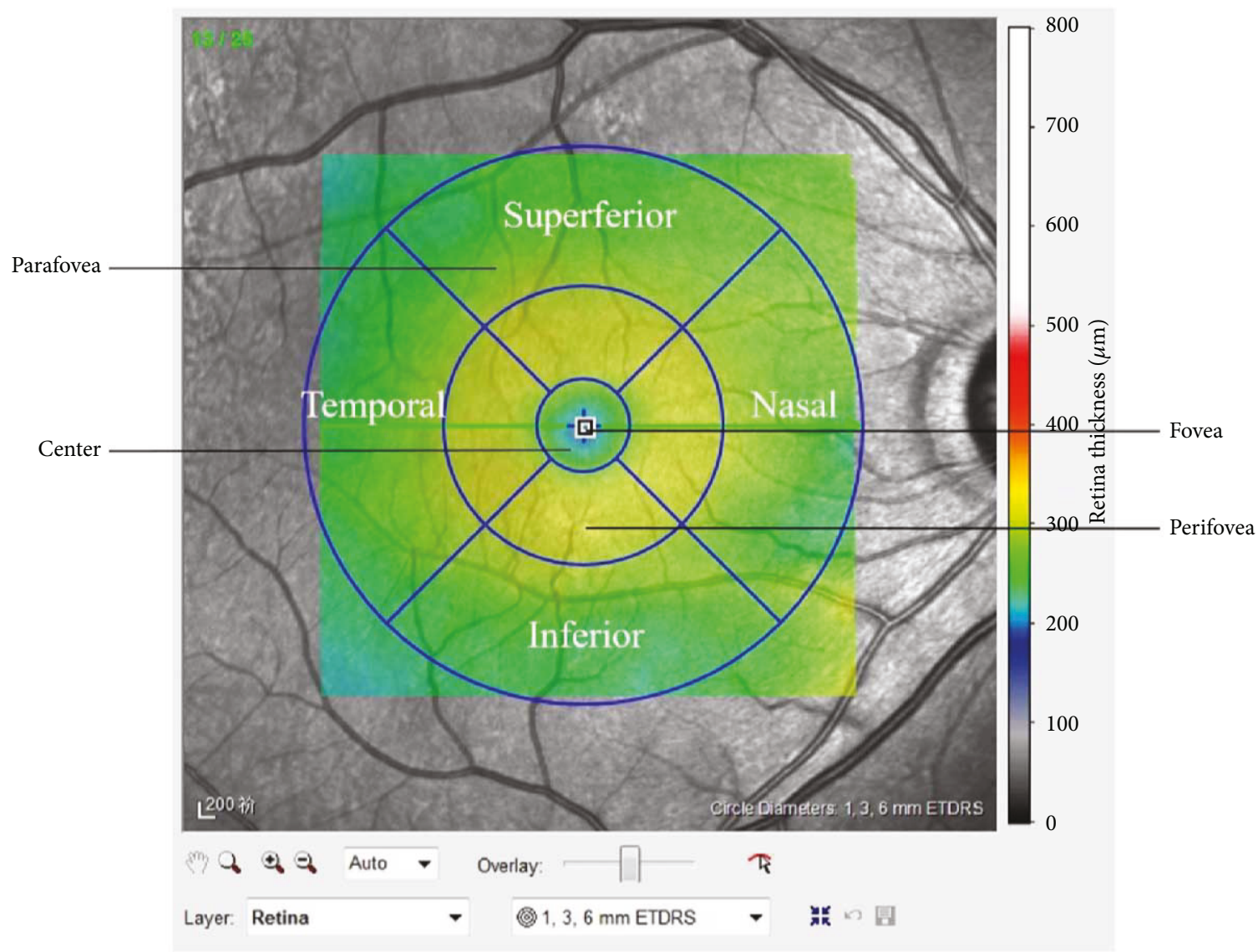

Figure 2: The ten areas examined included the fovea, central foveal circle (diameter $=1 \mathrm{~mm})$, parafoveal circle $($ diameter $=3 \mathrm{~mm}$ ), and perifoveal circle (diameter $=6 \mathrm{~mm}$ ). The parafoveal area and the perifoveal area were further subdivided into superior, inferior, temporal, and nasal subfields.

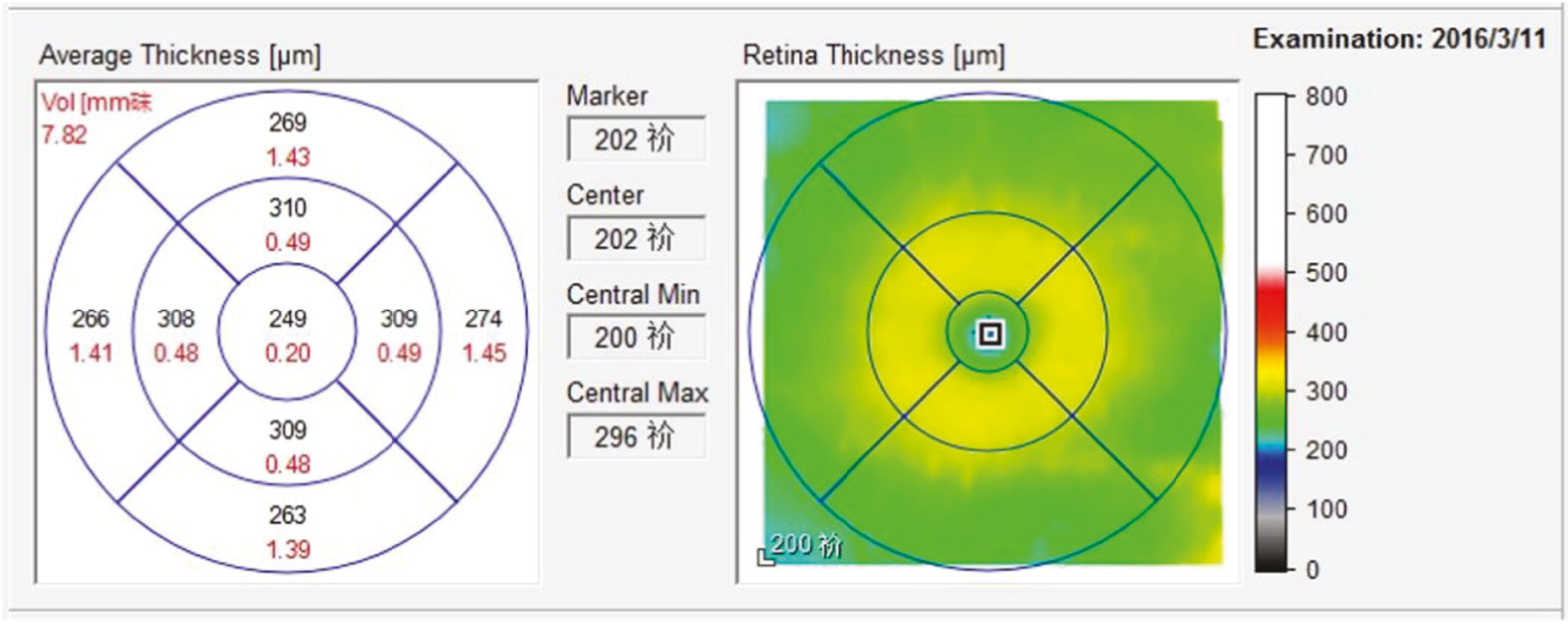

FIGURE 3: Retinal thickness map, in which averages of retinal thickness measurements of nine areas (except the fovea) are calculated and automatically displayed.

commonly decreased in diabetes, especially in the foveal and temporal (parafovea and perifovea) areas. We believe that these conclusions that differ from those of the above studies may have been associated with differences in the type of diabetes or degree of DR in subjects, the scan protocols, the measuring instruments used, and the patients' age and sex. The decreased retinal thicknesses in individuals with earlystage diabetes reflected neurodegenerative changes, such as 
TABLe 1: Clinical characteristics of the two groups.

\begin{tabular}{lccc}
\hline Clinical variables & $\begin{array}{c}\text { Diabetes (no DR) } \\
\text { Group A }(n=43)\end{array}$ & Control (normal) & Group B $(n=43)$ \\
\hline Age (years) & $64.4 \pm 8.4$ & $63.7 \pm 5.3$ & 0.65 \\
Male/female & $17 / 26$ & $15 / 28$ & 0.66 \\
History of hypertension & $14 / 43$ & $12 / 43$ & 0.64 \\
Duration of DM & $13.3 \pm 8.5$ & N/A & - \\
HbAlc level (\%) & $9.3 \pm 2.2$ & $5.3 \pm 1.0$ & $<\mathbf{0 . 0 0 1}$ \\
\hline
\end{tabular}

DR: diabetic retinopathy; DM: diabetes mellitus; HbAlc: glycosylated haemoglobin.

TABLE 2: Retinal thickness of ten areas in the two groups ( $\mu \mathrm{m}$, mean $\pm \mathrm{SD})$.

\begin{tabular}{lccc}
\hline Retinal areas & $\begin{array}{c}\text { Diabetes (no DR) } \\
\text { Group A }(n=86)\end{array}$ & Control (normal) & Group B $(n=86)$ \\
\hline Fovea & $215.8 \pm 18.9$ & $222.0 \pm 18.6$ & $\mathbf{0 . 0 4}$ \\
Center & $258.4 \pm 23.0$ & $261.3 \pm 16.8$ & 0.36 \\
Superior parafovea & $332.9 \pm 18.6$ & $337.3 \pm 15.0$ & 0.10 \\
Superior perifovea & $292.1 \pm 17.5$ & $296.0 \pm 14.6$ & 0.12 \\
Inferior parafovea & $328.6 \pm 16.4$ & $333.0 \pm 16.7$ & 0.09 \\
Inferior perifovea & $279.4 \pm 17.2$ & $282.9 \pm 15.1$ & 0.17 \\
Nasal parafovea & $334.3 \pm 18.5$ & $338.2 \pm 15.8$ & 0.15 \\
Nasal perifovea & $307.4 \pm 19.6$ & $311.8 \pm 17.4$ & 0.13 \\
Temporal parafovea & $319.9 \pm 16.7$ & $326.0 \pm 14.4$ & $\mathbf{0 . 0 1}$ \\
Temporal perifovea & $276.4 \pm 27.9$ & $284.8 \pm 17.4$ & $\mathbf{0 . 0 2}$ \\
\hline
\end{tabular}

SD: standard deviation; DR: diabetic retinopathy.

TABLE 3: Association between HbA1c levels and regions.

\begin{tabular}{|c|c|c|c|c|}
\hline \multirow{3}{*}{ Retinal areas } & \multicolumn{4}{|c|}{ Diabetes group only } \\
\hline & \multicolumn{2}{|c|}{$\mathrm{G}_{\mathrm{A} 1}$} & \multicolumn{2}{|c|}{$\mathrm{G}_{\mathrm{A} 2}$} \\
\hline & $r$ & $p$ & $r$ & $p$ \\
\hline Fovea & -0.15 & 0.35 & -0.30 & 0.09 \\
\hline Temporal parafovea & -0.16 & 0.33 & -0.12 & 0.06 \\
\hline Temporal perifovea & -0.09 & 0.57 & -0.31 & 0.04 \\
\hline
\end{tabular}

HbA1c: glycosylated haemoglobin.

loss or degeneration of glial cells, in diabetic retinas. Srinivasan et al. have observed decreased thicknesses in the parafovea and perifovea in diabetic patients with peripheral neuropathy [19]. As the disease progressed, neurodegeneration may have caused vascular permeability, which may have led to the thickening of the retinal layers.

The pathological mechanisms of DR are complicated and multifactorial. Vascular dysfunction, the basic pathology underlying diabetes, can occur in early stages of DR, in the absence of structural and functional abnormalities of the retina. The fovea has the highest density of cones and therefore has an increased metabolic demand [20]. The temporal region has the thinnest retina [21]. We hypothesize that the foveal and temporal regions may therefore be more susceptible to microvascular or ischaemic insults because of the above structural features.

Previous studies have reported that several risk factors, including $\mathrm{HbA} 1 \mathrm{c}$ level, duration of diabetes, hypertension, hyperlipidaemia, body mass index (BMI), sex, and insulin treatment, are involved in the development of DR. Among the factors above, a long duration of diabetes, high HbAlc levels, and insulin treatment are predictive factors for the deterioration of DR $[22,23]$. To determine the cause of retinal thinning in this study, we analyzed the relationships among $\mathrm{HbA} 1 \mathrm{c}$ level, duration of diabetes, and retinal thickness. Although the thickness of the temporal areas had a negative correlation with diabetes duration, the difference was not statistically significant. The reasons for this result may be that the course time provided by patients was the duration confirmed by diagnostic examination, which was often shorter than the actual duration of illness, and the so-called "course of diabetes" may not have been sufficient to lead to significative retinal structure damage.

$\mathrm{HbAlc}$ levels predict the incidence and progression of DR [24]. Retinal thickness is inversely correlated with HbA1c levels [25]. Another study has shown that a decrease in $\mathrm{HbAlc}$ is associated with thickening of inner retinal layers in the parafovea after bariatric surgery, which dramatically improves the metabolic profile in diabetes [26]. Hypoxic apoptosis of ganglion and axonal cells caused by the high affinity of HbAlc on oxygen (most oxygen molecule integrated with the elevated level of $\mathrm{HbAlc}$ instead of ganglion and axonal cells, which lead to the latter hypoxia) may be the most important underlying pathological mechanism. In this study, we also observed an inverse correlation between retinal thickness (temporal perifoveal area) and HbA1c levels. Our study indicated a strong correlation between DR and HbAlc. 
In summary, we observed that retinal thickness in the foveal and temporal areas was decreased in diabetes without any clinical signs of DR, and a high $\mathrm{HbAlc}$ level was associated with a thinner retina in the temporal perifoveal area in this study. Our results suggest that the neurodegenerative damage of the retina may occur before vasculopathy in the very early stages of diabetes. SD-OCT is a valuable diagnostic tool for providing detailed measurements of the retina for detection of early-stage DR. The limitations of this study included the predominantly homogenous subject group (100\% Asians from China), a self-reported duration of disease, and a relatively small sample size, which may have increased the bias of the study. Larger and longitudinal studies are necessary to verify these results.

\section{Conflicts of Interest}

None of the authors have any financial/conflicting interests to disclose.

\section{Authors' Contributions}

Jing Jiang was the first author of this study, and Jing Jiang and Yan Liu contributed equally to this work.

\section{Acknowledgments}

The research was supported by the National Nature Science Foundation of China (81670868) and Shanghai Science and Technology Committee Foundation (17DZ2260100).

\section{References}

[1] W. Rathmann and G. Giani, "Global prevalence of diabetes: estimates for the year 2000 and projections for 2030," Diabetes Care, vol. 27, no. 10, pp. 2568-2569, 2004.

[2] L. M. Ruta, D. J. Magliano, R. Lemesurier, H. R. Taylor, P. Z. Zimmet, and J. E. Shaw, "Prevalence of diabetic retinopathy in type 2 diabetes in developing and developed countries," Diabetic Medicine, vol. 30, no. 4, pp. 387-398, 2013.

[3] H. Sanchez-Tocino, A. Alvarez-Vidal, M. J. Maldonado, J. Moreno-Montanes, and A. Garcia-Layana, "Retinal thickness study with optical coherence tomography in patients with diabetes," Investigative Ophthalmology \& Visual Science, vol. 43, no. 5, pp. 1588-1594, 2002.

[4] R. Bafiq, R. Mathew, E. Pearce et al., "Age, sex, and ethnic variations in inner and outer retinal and choroidal thickness on spectral-domain optical coherence tomography," American Journal of Ophthalmology, vol. 160, no. 5, pp. 1034-1043.e1, 2015.

[5] F. D. Cabrera, H. M. Salinas, and C. A. Puliafito, "Automated detection of retinal layer structures on optical coherence tomography images," Optics Express, vol. 13, no. 25, pp. 10200-10216, 2005.

[6] S. C. Ozdek, M. A. Erdinc, G. Gurelik, B. Aydin, U. Bahceci, and B. Hasanreisoglu, "Optical coherence tomographic assessment of diabetic macular edema: comparison with fluorescein angiographic and clinical findings," Ophthalmologica, vol. 219, no. 2, pp. 86-92, 2005.

[7] K. V. Chalam, S. B. Bressler, A. R. Edwards et al., "Retinal thickness in people with diabetes and minimal or no diabetic retinopathy: Heidelberg Spectralis optical coherence tomography," Investigative Ophthalmology \& Visual Science, vol. 53, no. 13, pp. 8154-8161, 2012.

[8] H. Y. Cho, D. H. Lee, S. E. Chung, and S. W. Kang, "Diabetic retinopathy and peripapillary retinal thickness," Korean Journal of Ophthalmology, vol. 24, no. 1, pp. 16-22, 2010.

[9] S. Srinivasan, N. Pritchard, G. P. Sampson et al., "Retinal thickness profile of individuals with diabetes," Ophthalmic \& Physiological Optics, vol. 36, no. 2, pp. 158-166, 2016.

[10] Y. Bao, X. Ma, H. Li et al., "Glycated haemoglobin A1c for diagnosing diabetes in Chinese population: cross sectional epidemiological survey," $B M J$, vol. 340 , no. may17 1, article c2249, 2010.

[11] C. Liu, C. Yang, Y. Zhao et al., "Associations between longterm exposure to ambient particulate air pollution and type 2 diabetes prevalence, blood glucose and glycosylated hemoglobin levels in China," Environment International, vol. 92-93, pp. 416-421, 2016.

[12] W. W. Harrison, A. Chang, M. G. Cardenas et al., "Blood pressure, vessel caliber, and retinal thickness in diabetes," Optometry and Vision Science, vol. 89, no. 12, pp. 17151720, 2012.

[13] Y. Wu, Y. Ding, Y. Tanaka, and W. Zhang, "Risk factors contributing to type 2 diabetes and recent advances in the treatment and prevention," International Journal of Medical Sciences, vol. 11, no. 11, pp. 1185-1200, 2014.

[14] S. Srinivasan, N. Pritchard, G. P. Sampson et al., "Retinal tissue thickness in type 1 and type 2 diabetes," Clinical \& Experimental Optometry, vol. 99, no. 1, pp. 78-83, 2016.

[15] M. Demir, E. Oba, B. Dirim, E. Ozdal, and E. Can, "Retracted article: central macular thickness in patients with type 2 diabetes mellitus without clinical retinopathy," BMC Ophthalmology, vol. 13, no. 1, p. 11, 2013.

[16] Y. Chen, J. Li, Y. Yan, and X. Shen, "Diabetic macular morphology changes may occur in the early stage of diabetes," BMC Ophthalmology, vol. 16, no. 1, p. 12, 2016.

[17] I. Pires, A. R. Santos, S. Nunes, and C. Lobo, "Macular thickness measured by stratus optical coherence tomography in patients with diabetes type 2 and mild nonproliferative retinopathy without clinical evidence of macular edema," Ophthalmologica, vol. 229, no. 4, pp. 181-186, 2013.

[18] H. Y. Park, I. T. Kim, and C. K. Park, "Early diabetic changes in the nerve fibre layer at the macula detected by spectral domain optical coherence tomography," The British Journal of Ophthalmology, vol. 95, no. 9, pp. 1223-1228, 2011.

[19] S. Srinivasan, N. Pritchard, D. Vagenas et al., "Retinal tissue thickness is reduced in diabetic peripheral neuropathy," Current Eye Research, vol. 41, no. 10, pp. 1359-1366, 2016.

[20] E. Aguilar, M. Friedlander, and R. F. Gariano, "Endothelial proliferation in diabetic retinal microaneurysms," Archives of Ophthalmology, vol. 121, no. 5, pp. 740-741, 2003.

[21] A. Chan, J. S. Duker, T. H. Ko, J. G. Fujimoto, and J. S. Schuman, "Normal macular thickness measurements in healthy eyes using stratus optical coherence tomography," Archives of Ophthalmology, vol. 124, no. 2, pp. 193-198, 2006.

[22] A. Chorny, T. Lifshits, A. Kratz et al., "Prevalence and risk factors for diabetic retinopathy in type 2 diabetes patients in Jewish and Bedouin populations in southern Israel," Harefuah, vol. 150, no. 12, pp. 906-10, 935, 2011. 
[23] Z. P. Yan and J. X. Ma, "Risk factors for diabetic retinopathy in northern Chinese patients with type 2 diabetes mellitus," International Journal of Ophthalmology, vol. 9, no. 8, pp. 1194-1199, 2016.

[24] J. W. Yau, S. L. Rogers, R. Kawasaki et al., "Global prevalence and major risk factors of diabetic retinopathy," Diabetes Care, vol. 35, no. 3, pp. 556-564, 2012.

[25] B. Asefzadeh, B. M. Fisch, C. E. Parenteau, and A. A. Cavallerano, "Macular thickness and systemic markers for diabetes in individuals with no or mild diabetic retinopathy," Clinical \& Experimental Ophthalmology, vol. 36, no. 5, pp. 455-463, 2008.

[26] T. Brynskov, C. S. Laugesen, A. K. Floyd, and T. L. Sorensen, "Thickening of inner retinal layers in the parafovea after bariatric surgery in patients with type 2 diabetes," Acta Ophthalmologica, vol. 94, no. 7, pp. 668-674, 2016. 


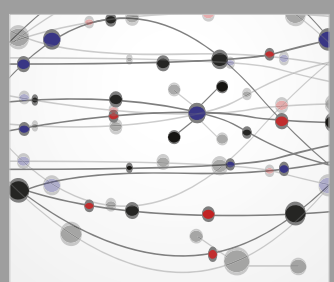

The Scientific World Journal
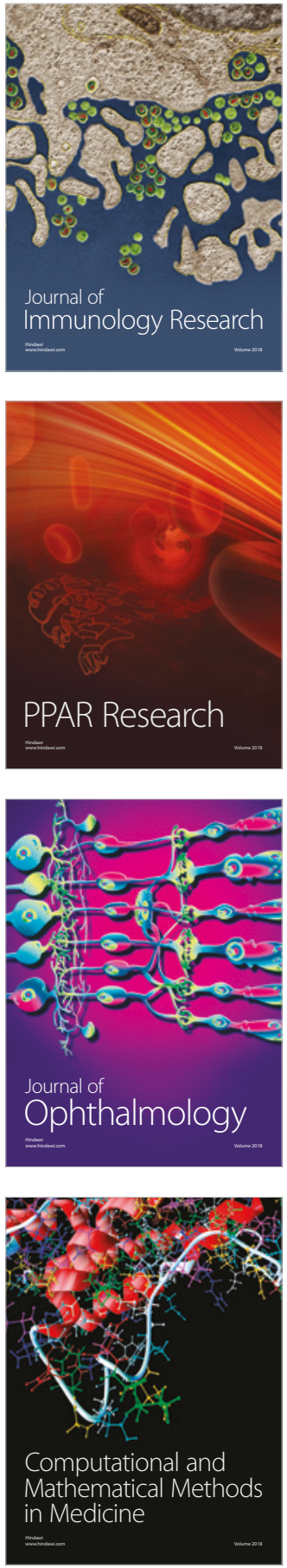

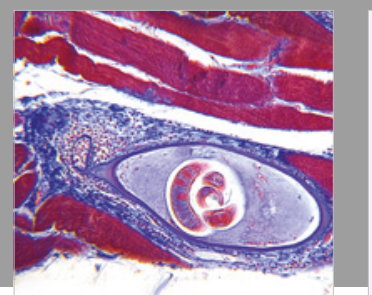

Gastroenterology Research and Practice

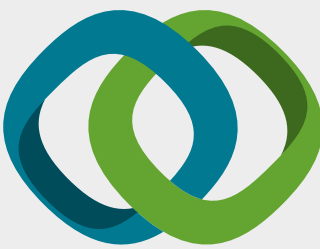

\section{Hindawi}

Submit your manuscripts at

www.hindawi.com
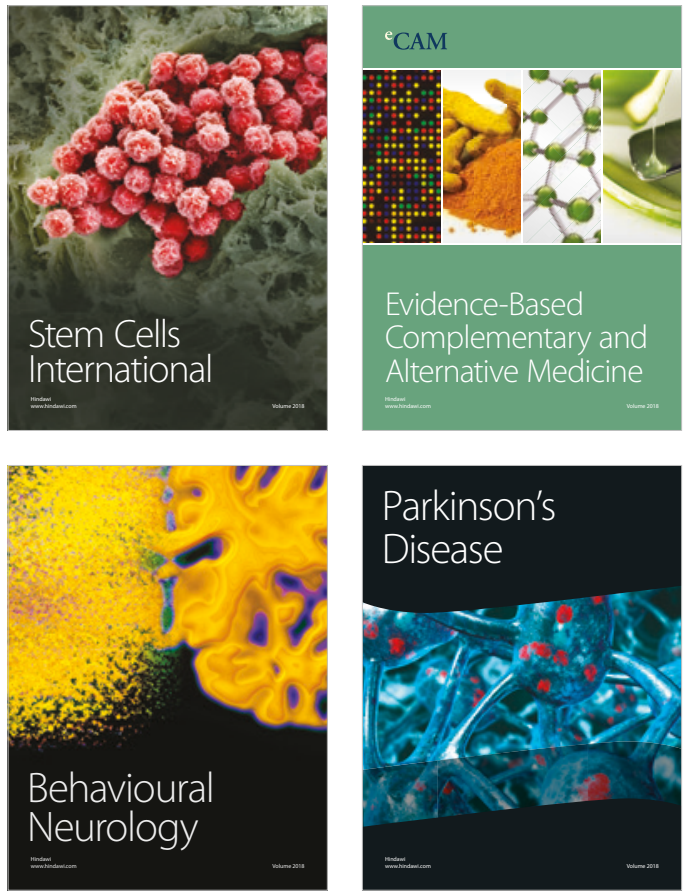

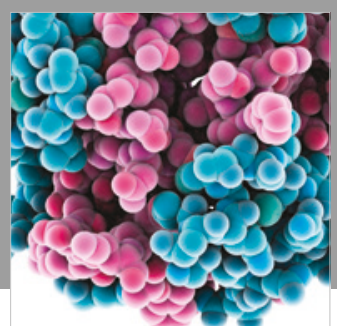

ournal of

Diabetes Research

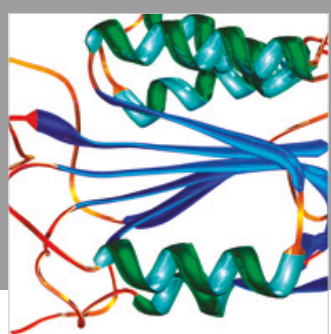

Disease Markers
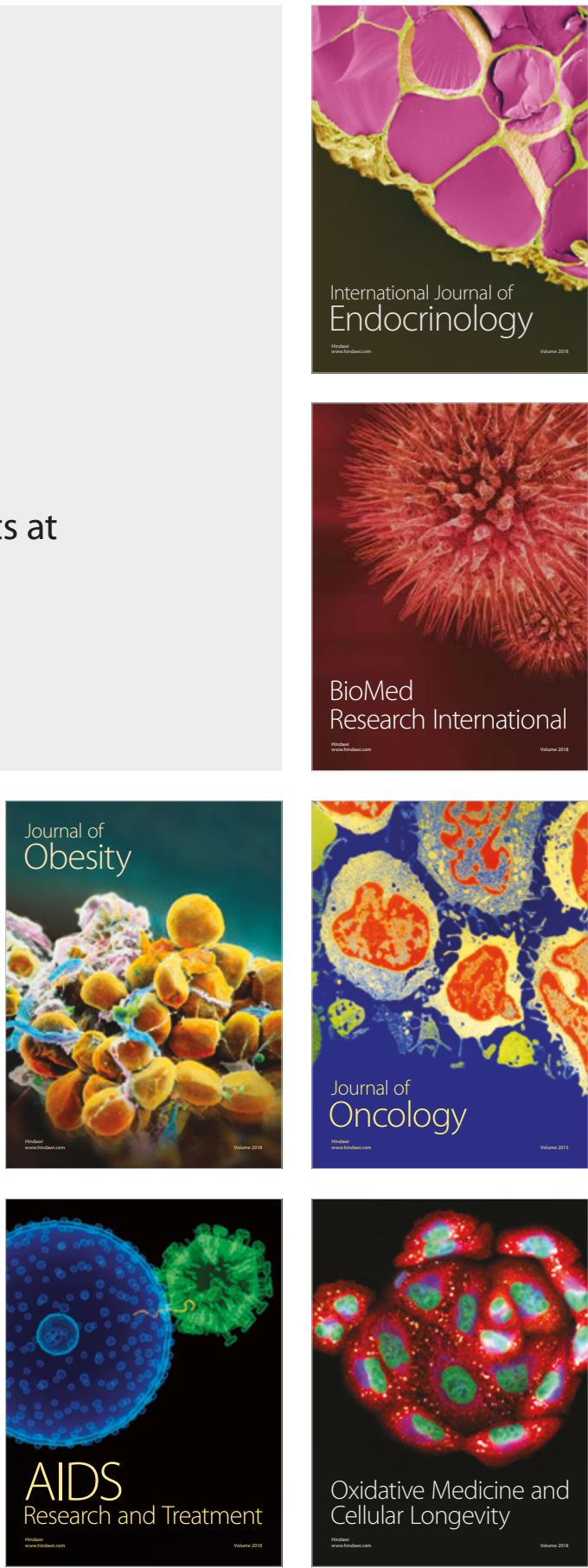